\title{
Automatic Segmentation of the Pulmonary Lobes from Fissures, Airways, and Lung Borders: Evaluation of Robustness against Missing Data
}

\author{
Eva M. van Rikxoort, Mathias Prokop, Bartjan de Hoop, Max A. Viergever, \\ Josien P.W. Pluim, and Bram van Ginneken
}

Image Sciences Institute, University Medical Center Utrecht, The Netherlands

\begin{abstract}
Automatic segmentation of structures with missing or invisible borders is a challenging task. Since structures in the lungs are related, humans use contextual and shape information to infer the position of invisible borders. An example of a task in which the borders are often incomplete or invisible is the segmentation of the pulmonary lobes. In this paper, a fully automatic segmentation of the pulmonary lobes in chest CT scans is presented. The method is especially designed to be robust to incomplete fissures by incorporating contextual information from automatic lung, fissure, and bronchial tree segmentations, as well as shape information. Since the method relies on the result of automatic segmentations, it is important that the method is robust against failure of one or more of these segmentation methods. In an extensive experiment on 10 chest CT scans with manual segmentations, the robustness of the method to incomplete fissures and missing input segmentations is shown. In a second experiment on 100 chest CT scans with incomplete fissures, the method is shown to perform well.
\end{abstract}

\section{Introduction}

In medical images, structures are often incomplete, missing, or barely visible, so that they cannot be detected reliably by computer algorithms. In the lungs, anatomical structures are related; the position and shape of one anatomical structure provides information about the possible positions and shapes of other anatomical structures. Therefore, when the borders of structures in the lungs are not visible, human experts infer the position of the borders using anatomical cues in a flexible manner.

An example of a segmentation task for which the borders are often missing or hard to detect automatically is the segmentation of the pulmonary lobes. The human lungs are divided into five lobes, there are three lobes in the right lung and two in the left lung. The physical border between the lobes are the pulmonary fissures, the major fissures separate the upper and lower lobes in both lungs, the minor fissure in the right lung separates the middle lobe from the 
upper lobe. Several studies have shown that incomplete pulmonary fissures are a very frequent phenomenon [1]. In this work we present a method for automatic segmentation of the pulmonary lobes from computed tomography (CT) scans. Our method combines multiple anatomical cues to arrive at a segmentation, in an attempt to mimic the flexibility of human image understanding. A few papers concerning segmentation of pulmonary lobes have been published [2, 3], 4]. However, none of those papers present a completely automatic method for lobe segmentation in cases with incomplete fissures. In [4, a lobe segmentation in cases with incomplete fissures is presented that requires manual interaction in $25 \%$ of the cases.

Since fissures are often incomplete or hard to locate automatically, deriving a lobe segmentation from a fissure segmentation is not trivial. There are several alternatives that can be used to infer the lobe borders. Important information can be extracted from the segmentation of the lungs since the shape of the lobes is constrained by the shape of the lungs. In addition, the topology of the bronchial tree can be exploited. The different lobes are separately supplied by the first subdivisions of the bronchial tree after the main bronchi. Therefore, given a division of the bronchial tree into its lobar bronchi, a segmentation of the pulmonary lobes can be approximated by assigning each voxel inside the lungs to the lobar label of the nearest bronchus. Next to anatomical information, knowledge about the shape of the various lobes can be used. Radiologists combine all this information when they infer the lobar boundary in a CT scan.

We present a multi-atlas approach in which existing lobar segmentations are deformed to unseen test scans in which the fissures, as far as possible, the lungs, and the airway tree have been segmented. The key element of our method is the design of a cost function that exploits information from fissures, airway tree, and lung border in an effective way, such that less reliable information (lungs, airways) is only used when the most reliable information (fissures) is missing. To cope with the anatomical variation in the shapes of the lobes, an atlas selection mechanism is introduced.

The proposed method is fairly complex, in the sense that it requires the output of three segmentation algorithms as input. Each of these three tasks is not trivial by itself, therefore, it is likely that they will fail occasionally. Moreover, the most important information, derived from the detection of fissures, is often incomplete due to fissural incompleteness. Therefore, we carefully evaluate in this paper how robust the proposed method is to fissural incompleteness and missing lung, airway tree, and fissure segmentations. This also serves as a validation of the rationale of our approach: if these three structures in concert are indeed all important to infer the position of the lobar borders, leaving out any of this information should decrease the performance of the method. In an additional experiment on 100 chest CT scans, the performance of the method is evaluated. 


\section{Method}

\subsection{Overview}

A multi-atlas lobe segmentation method was developed in which information from scans with complete fissures is transformed to a test scan to obtain an anatomically plausible lobe segmentation in the test scan. Since registering chest CT scans from different subjects directly does not lead to satisfactory results due to anatomical variations, anatomical information obtained from automatic segmentations of the lungs, the fissures, and the bronchial tree are employed during registration. The method requires a set of atlases with segmentations of the lung borders, fissures, and lobes to be available (Section 3). For a test image, the method consists of the following 5 steps: 1) the lung borders, fissures, and airway tree are segmented. 2) the most appropriate atlas is automatically selected. 3) the most appropriate atlas is registered to the test scan. 4) the lobe segmentation is propagated. 5) the result is post processed.

\subsection{Prerequisite Segmentations}

The method starts by segmenting the lung borders, fissures, and approximated lobe borders from the bronchial tree in the test image. The lung, fissure, and bronchial tree segmentations applied in this paper are all based on previous work $([5], 6]$, [] $)$ and are therefore not described here. From the segmentation of the lungs, the lung borders are extracted as those voxels in the lung segmentation for which one of the 8-connected neighbors is outside the lung segmentation. The segmentation of the bronchial tree is used to approximate the lobe borders. The method described by [7] provides the anatomical labels for airway branches up to segmental level. From these anatomical labels, the lobar label of each airway segment is know. Given this labeled airway, the lobes can be approximated by assigning each voxel in the lungs to the lobar label of the closest bronchi. Approximated lobar boundaries are extracted as those voxels for which one of the 8 -connected neighbors in the lungs belongs to another lobe. The approximated lobe borders are illustrated in Figure 1.
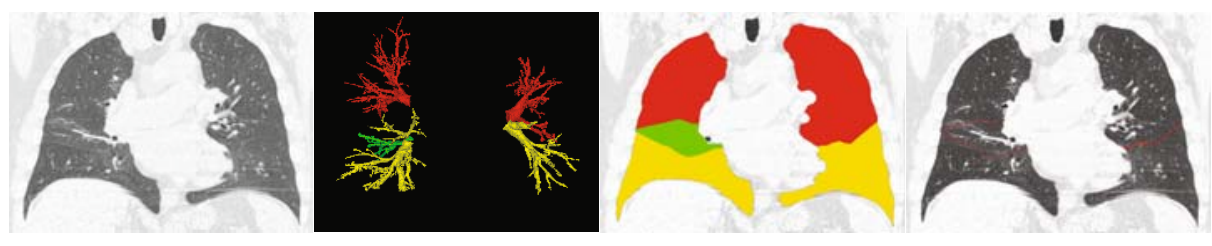

Fig. 1. Illustration of the approximated lobe borders from the bronchial tree. The frames show the original slice, a rendering of the airways, the approximated lobes, and the approximated lobe borders, respectively. 


\subsection{Multi-atlas Lobe Segmentation}

For the multi-atlas lobe segmentation it is assumed that a set of $n$ atlases with complete pulmonary fissures and corresponding segmented lungs, fissures, and lobes are available (Section 31). For a test scan in which the lungs, fissures, and airways have been automatically segmented, the following steps are performed: 1) the test image and atlases are prepared for registration. 2) all atlases are registered to the test scan using a fast registration and the most promising atlas is selected for further processing. 3) the lobes in the test scan are segmented by accurately registering the selected atlas and propagating the lobe labels with the resulting transformation, followed by a postprocessing.

Registration. In this paper elastix[1 version 3.9 was used for registration. The atlas-based lobe segmentation requires two registration methods to be available: a fast (computationally cheap) method which is used to select the atlas that is most similar to the test image, and an accurate (computationally expensive) method which transforms an atlas image to a target image with high accuracy for the final lobe segmentation. For both the fast and the accurate registration SSD was used as a similarity measure. The images were first roughly aligned with an affine transformation, followed by a non-rigid registration modeled by Bsplines. The difference between the fast and accurate registration is the number of iterations used per resolution for the non-rigid registration: five resolutions were used, the fast registration performed 100 iterations per resolution, the accurate registration performed 600 iterations per resolution.

Input to Registration. The registration is performed for two images simultaneously: one image containing the lung border and the other image containing the fissure segmentations. The cost function is calculated as a combination of both registrations, giving them both equal weight. Since registration involves an optimization towards a minimum of the similarity measure, not the binary segmentations are used as input but the distance transform to those segmentations. This smoothes the cost function and ensures that the method is gradually attracted to the correct position. The distance transforms to the different structures are cut off at $14 \mathrm{~mm}$. For the test image, the fissure segmentations are augmented with the approximated lobe border from the bronchial tree only on positions where the fissures are not defined. This is achieved as follows: The distance transforms to the fissures and the approximated lobe borders are calculated separately. Next, the values of the distance transform to the approximated lobe border are added to the distance transform of the fissures only on those positions where the fissure distance transform is not defined.

Since SSD is used as a cost function, different structures can be given different weights during registration by multiplying their values by a weighing factor. For the lobe segmentation, three parameters are introduced controlling the weights of the different structures: $\omega_{f}$ for the fissure, $\omega_{l}$ for the lung border, and $\omega_{b}$ for the bronchial tree. The appropriate values for the different weights were found

${ }^{1}$ elastix is publicly available at http://elastix.isi.uu.nl. 
to be $\omega_{f}=4, \omega_{l}=1$, and $\omega_{b}=1$. This setting ensures that the fissures, when available, are the most important information used during the registration.

Atlas Selection. An atlas selection is performed in which the atlas that is anatomically most similar to the test scan is automatically selected. For this atlas selection, all atlases are registered to the test scan using fast registration, and the fissure segmentation of the atlases are transformed. The most promising atlas for the lobe segmentation of a particular test scan is the scan for which the fissures of the transformed scan line up best with the fissures of the test scan. The atlas with the highest number of voxels of the transformed fissure within 1 $\mathrm{mm}$ of the fissure in the test scan is selected to be the most promising atlas for further processing.

Lobe Segmentation. The lobes in the test scan are now segmented by registering the selected atlas to the test scan using accurate registration, and transforming the lobe segmentation available for the atlas to the test image. This results in a lobe segmentation for the test image. Since the lung borders of the atlas and test image are not forced to line up during the registration, a post processing is necessary. Every voxel inside the lung segmentation of the test image that is not assigned to a lobe after the transformation of the atlas lobes, is given the label of the closest voxel in the transformed lobe segmentation.

\section{Materials and Experiments}

Atlases. A set of five atlases with complete pulmonary fissures was taken from a lung cancer screening trial with low dose CT [8]. The lungs and fissures in the atlases were automatically segmented. The results of the automatic methods were visually checked and edited where needed by a human observer. Since the fissures in the test scans were complete, a lobe segmentation was extracted directly from the segmented fissures.

Test Data. Two test sets have been used in this paper, which we will refer to as test set A and test set B. Test set A contains 10 normal dose $(120 \mathrm{kV}, 100$ to 150 $\mathrm{mAs}$ ) inspiration CT chest scans of 10 different patients. The scans were selected to show (nearly) complete fissures in one of the lungs (visually confirmed). A human observer manually indicated the fissures in every fourth coronal slice. Test set B contains 100 low dose scans, selected by visual inspection to contain substantially incomplete fissures. All scans were reconstructed to $512 \times 512$ matrices, slice thickness $1 \mathrm{~mm}$. To evaluate the performance and robustness of the automatic lobe segmentation three different experiments were performed:

1) To quantify the robustness of the automatic lobe segmentation to different levels of incomplete pulmonary fissures, an evaluation was performed on the scans from test set A. An experiment was setup in which parts of fissures were automatically removed to simulate the fissures being incomplete, for each lung, for each fissure separately. For a scan with complete fissures, the fissure was automatically segmented. Next, a part of the fissure was automatically removed from the hilum inward to mimic a scan with an incomplete fissure. Since the lobe 
Table 1. Mean distance $(\mathrm{mm})$ between the manually drawn lobe border (complete fissure) and the automatically found lobe border for different percentages of the fissure removed, for different settings of the weights $\omega_{f}, \omega_{l}$, and $\omega_{b}$

\begin{tabular}{|c|c|c|c|c|c|c|c|c|}
\hline \multirow[t]{2}{*}{$\omega_{f}-\omega_{l}-\omega_{b}$} & \multirow[t]{2}{*}{ setting } & \multicolumn{7}{|c|}{$0 \% 10 \% 20 \% 30 \% 40 \% 50 \%$ 60\% $70 \%$} \\
\hline & & \multicolumn{7}{|c|}{ Left major fissure } \\
\hline$\overline{4-1-1}$ & all structures & $\begin{array}{|ll|}0.51 & 0.54 \\
\end{array}$ & $\overline{0.63}$ & 0.70 & 0.93 & 1.10 & 1.58 & 1.81 \\
\hline $4-1-0$ & no airways & $0.52 \quad 0.59$ & 0.83 & 0.94 & 1.17 & 1.64 & 3.02 & 3.94 \\
\hline $4-0-1$ & no lungs & $0.48 \quad 0.56$ & 0.67 & 0.73 & 0.99 & 1.27 & 1.68 & 1.99 \\
\hline $0-1-1$ & no fissures & $4.52 \quad 4.52$ & 4.52 & 4.52 & 4.52 & 4.52 & 4.52 & 4.52 \\
\hline $4-0-0$ & only fissures & 0.490 .57 & 0.84 & 1.06 & 1.31 & 1.89 & 3.73 & 5.47 \\
\hline $0-1-0$ & only lungs & $5.47 \quad 5.47$ & 5.47 & 5.47 & 5.47 & 5.47 & 5.47 & 5.47 \\
\hline \multirow[t]{2}{*}{$0-0-1$} & only airways & $5.28 \quad 5.28$ & 5.28 & 5.28 & 5.28 & 5.28 & 5.28 & 5.28 \\
\hline & & \multicolumn{7}{|c|}{ Right major fissure } \\
\hline 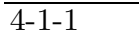 & actures & 1.221 .32 & 1.54 & 1.79 & 2.18 & 2.56 & 3.22 & 3.71 \\
\hline $4-1-0$ & no airways & $1.25 \quad 1.39$ & 1.70 & 2.13 & 2.83 & 3.76 & 5.28 & 7.31 \\
\hline $4-0-1$ & no 1 & $1.21 \quad 1.34$ & 1.57 & 1.78 & 2.08 & 2.58 & 3.31 & 3.90 \\
\hline $0-1-1$ & no fissures & $6.33 \quad 6.33$ & 6.33 & 6.33 & 6.33 & 6.33 & 6.33 & 6.33 \\
\hline $4-0-0$ & only fissures & $1.25 \quad 1.41$ & 1.74 & 2.15 & 2.70 & 3.66 & 5.41 & 7.52 \\
\hline $0-1-0$ & only & 6.626 .62 & 6.62 & 6.62 & 6.62 & 6.62 & 6.62 & 6.62 \\
\hline \multirow[t]{2}{*}{$0-0-1$} & only airways & $6.31 \quad 6.31$ & 6.31 & 6.31 & 6.31 & 6.31 & 6.31 & 6.31 \\
\hline & & \multicolumn{7}{|c|}{ Right minor fissure } \\
\hline $4-1-1$ & all structures & $\begin{array}{ll}1.08 & 1.02\end{array}$ & 1.37 & 2.00 & 2.60 & 3.38 & 4.61 & 5.90 \\
\hline $4-1-0$ & no airways & 1.081 .07 & 1.61 & 2.30 & 3.21 & 4.15 & 6.56 & 8.06 \\
\hline $4-0-1$ & no lungs & $1.06 \quad 1.02$ & 1.38 & 1.98 & 2.68 & 3.40 & 4.81 & 6.11 \\
\hline $0-1-1$ & no fissures & $6.23 \quad 6.23$ & 6.23 & 6.23 & 6.23 & 6.23 & 6.23 & 6.23 \\
\hline $4-0-0$ & only fissures & $1.07 \quad 1.04$ & 1.58 & 2.24 & 3.03 & 4.05 & 6.60 & 8.20 \\
\hline $0-1-0$ & only lungs & $6.93 \quad 6.93$ & 6.93 & 6.93 & 6.93 & 6.93 & 6.93 & 6.93 \\
\hline $0-0-1$ & only airways & $6.23 \quad 6.23$ & 6.23 & 6.23 & 6.23 & 6.23 & 6.23 & 6.23 \\
\hline
\end{tabular}

segmentation method does not use the original CT data, it does not benefit from the fact that the fissure is actually complete. For the experiment, $10,20, \ldots 70 \%$ of the fissures were removed. Both for the left and the right lung, six of the scans showed complete fissures and were used for this experiment. The mean distance between the manually defined fissures and the automatically determined lobe border was used as an evaluation measure.

2) To investigate the robustness of the method against missing lung, fissure, or airway segmentations, the first experiment was repeated using different settings for the weights $\omega_{f}, \omega_{l}$, and $\omega_{b}$. The settings used are given in Table 1. For example, setting $\omega_{f}=4, \omega_{l}=1, \omega_{b}=0$, does not take into account the approximated lobe border from the bronchial tree during the segmentation.

3) To evaluate the performance of the automatic lobe segmentation in a large set of scans, an observer study was setup in which a radiologist visually scored the performance of the lobe segmentation method for test set B. Each lobe border was scored to be correct, almost correct, or incorrect. 


\section{Results}

In Table 1 the results for the first and second experiment are provided. Example output of the lobe segmentation for different percentages of fissures removed are provided in Figure 2. The results of the observer study in test set B were as follows: for the left major fissure, $79 \%$ of the lobe borders were scored as correct, the other $21 \%$ as almost correct. For the right major fissure and right minor fissure those numbers are $89 \%$, and $11 \%$, and $76 \%$, and $22 \%$ respectively. For the right minor fissure two lobe borders were scored as incorrect. In both cases, the fissure was completely absent.

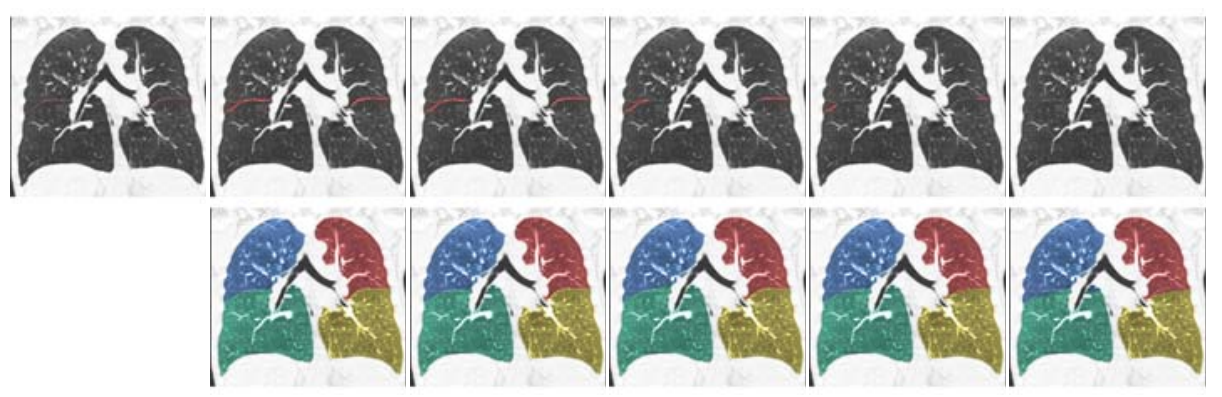

Fig. 2. Example output of the experiment in which parts of fissures were automatically removed to test the robustness of the automatic lobe segmentation against incomplete fissures. The first row shows the original image with the fissure segmentations, in the second row the resulting lobe segmentations are shown. The first column shows the ground truth. The next columns show the results for $0 \%, 10 \%, 30 \%, 50 \%$, and $70 \%$ of the fissures removed, respectively.

\section{Discussion and Conclusion}

Complete fissures are rare, which makes automatic lobe segmentation challenging. In this paper, a completely automatic lobe segmentation method robust against the presence of incomplete pulmonary fissures was presented. By using an atlas based approach in which information from scans with complete fissures are transformed to test scans with incomplete fissures, the fissures are extended in an anatomically plausible manner. The methodology applied in this paper is similar to the methodology humans use when segmenting lobes manually: when the fissures are complete, they define the lobe borders. When no fissure is present, knowledge about the shapes of the various lobes as well as anatomical information, such as the bronchial tree, are used.

The results of the first two experiments clearly show that the presented automatic lobe segmentation performs well in cases with incomplete fissures. From Table 1 it can be seen that even when $70 \%$ of the fissures were removed, the automatically extracted lobe border was still within $4 \mathrm{~mm}$ of the real lobe border for the major fissures and within $6 \mathrm{~mm}$ for the right minor fissure, which 
are clinically acceptable results. The accuracy of the automatic lobe segmentation gradually decreases when less fissures are present. In addition, the results shown in Figure 2 show that the lobe segmentation remains similar to the lobe segmentation with complete fissures even after removing $70 \%$ of the fissure.

The results in Table 1 also show the contribution of the different segmented structures used during registration. The first observation that can be made is that when the fissures are complete, they provide enough information to obtain an accurate lobe segmentation. Secondly, it can be seen that adding the approximated lobe border from the bronchial tree where fissures are incomplete leads to better performance: for all fissures, the performance decreases when $\omega_{b}$ is set to 0 . In addition, when only the approximated lobe border from the bronchial tree is used, the average error is still within $7 \mathrm{~mm}$ for each fissure, indicating that it is a reasonable approximation of the position of the true lobe border. From Table 1 it can also be appreciated that the method is in general robust against missing input segmentations.

Overall, using the fissures, lungs, and bronchial tree as input gave the best results. Which confirm the rational behind our method that combining information from the fissures, lungs, and airways leads to superior results. This setting was used for a visual evaluation in the 100 scans from test set B. This evaluation showed that a radiologist agreed with the automatic result in $81 \%$ of the cases on average, only for two cases, only for the minor fissure, the segmentation was judged as incorrect. It is important to realize the difficulty of the task of automatic lobe segmentation with incomplete fissures. The scans in test set B were selected to show substantially incomplete fissures. As a comparison, Ukil et al [4] performed a similar evaluation for scans with incomplete fissures in which $40 \%$ of the lobe segmentations were judged as correct.

To conclude, an automatic lobe segmentation method was presented that was specifically designed to be robust against incomplete fissures by incorporating contextual information from automatic segmentations of other anatomical structures. Next to good performance in cases with incomplete fissures, the method was shown to be able to handle missing input segmentations.

\section{References}

1. Aziz, A., Ashizawa, K., Nagaoki, K., Hayashi, K.: High resolution CT anatomy of the pulmonary fissures. Journal of Thoracic Imaging 19(3), 186-191 (2004)

2. Kuhnigk, J.M., Dicken, V., Zidowitz, S., Bornemann, L., Kuemmerlen, B., Krass, S., Peitgen, H.O., Yuval, S., Jend, H.H., Rau, W.S., Achenbach, T.: New tools for computer assistance in thoracic CT part 1. Functional analysis of lungs, lung lobes and bronchopulmonary segments. Radiographics 25(2), 525-536 (2005)

3. Zhang, L., Hoffman, E.A., Reinhardt, J.M.: Atlas-driven lung lobe segmentation in volumetric x-ray CT images. IEEE Transactions on Medical Imaging 25(1), 1-16 (2006)

4. Ukil, S., Reinhardt, J.M.: Anatomy-guided lung lobe segmentation in x-ray CT images. IEEE Transactions on Medical Imaging 28(2), 202-214 (2009) 
5. van Rikxoort, E.M., de Hoop, B., Viergever, M.A., Prokop, M., van Ginneken, B.: Automatic lung segmentation from thoracic ct scans using a hybrid approach with error detection. Medical Physics 36(7) (2009)

6. van Rikxoort, E.M., van Ginneken, B., Klik, M.A.J., Prokop, M.: Supervised enhancement filters: application to fissure detection in chest CT scans. IEEE Transactions on Medical Imaging 27(1), 1-10 (2008)

7. van Ginneken, B., Baggerman, W., van Rikxoort, E.M.: Robust segmentation and anatomical labeling of the airway tree from thoracic CT scans. In: Metaxas, D., Axel, L., Fichtinger, G., Székely, G. (eds.) MICCAI 2008, Part I. LNCS, vol. 5241, pp. 219-226. Springer, Heidelberg (2008)

8. Xu, D.M., Gietema, H., de Koning, H., Vernhout, R., Nackaerts, K., Prokop, M., Weenink, C., Lammers, J., Groen, H., Oudkerk, M., van Klaveren, R.: Nodule management protocol of the NELSON randomised lung cancer screening trial. Lung Cancer 54(2), 177-184 (2006) 